\title{
Load Distribution in Tooth and Implant-Abutment Identical Cases
}

\author{
Muhammed Mutahhar Ulusoy' (1), Hakan Arinc ${ }^{2}$ (D), Asiye Nehir Ozden ${ }^{3}$ (]) \\ 'Department of Prosthodontics, Near East University, Faculty of Dentistry, Nicosia/TRNC Mersin, Turkey \\ 2Department of Prosthodontics, Istanbul Okan University, Faculty of Dentistry, Tuzla Campus, Istanbul, Turkey \\ ${ }^{3}$ Department of Prosthodontics, Near East University, Faculty of Dentistry, Mersin, Turkey
}

ORCID iDs of the authors: M.M.U. 0000-0002-6090-5747; H.A. 0000-000I-8675-648X; A.N.O. 0000-000I-7084-7I33.

Cite this article as: Ulusoy MM, Arinc H, Ozden AN. Load Distribution in Tooth and Implant-Abutment Identical Cases. Cyprus J Med Sci 2020; 5(3): 2II-6.

\section{BACKGROUND/AIMS}

We sought to compare the biomechanical behavior of dental implants with that of natural teeth under identical situations in accordance with Ante's law.

\section{MATERIAL and METHODS}

We used finite element analysis to compare identical cases. We designed different combinations [tooth-supported models (TSMx) and implant-supported models (ISMx)] [TSM0: full-arch dentition, TSMI: 5-7 tooth-supported fixed dental prosthesis (FDP); TSM2: 4-7 toothsupported FDP; TSM3: 3-7 tooth-supported FDP; ISM0: full-arch dental implant-supported artificial crowns for each tooth; ISMI: 5-7 dental implant-supported FDP; ISM2: 4-7 dental implant-supported FDP; ISM3: and 3-7 dental implant-supported FDP]. We used Cobaltchromium supported ceramic as the prosthetic material. We used a foodstuff model to apply a $100 \mathrm{~N}$ of load for each tooth in the case of mastication.

\section{RESULTS}

In general, ISMs showed higher stress values than identical TSMs. The distribution of stress in the cortical bone was similar in identical models regardless of the abutment type. The maximum and minimum principal stress values in the cortical bone increased with the number of missing teeth. The trend in stress values was different between ISMs and TSMs.

\section{CONCLUSION}

Within the limitations of this study, stress distribution was similar in both abutment types. However, there was a difference in the magnitude and change in the magnitude of stress values of dental implants and tooth abutments. Our findings reveal that Ante's law may not be suitable as a guideline for dental implant treatment due to the difference in the trends of the maximum and minimum principal stress values.

Keywords: Cortical bone, dental abutments, dental implants, dental models, finite element analysis, mastication

\section{INTRODUCTION}

Implantation of partial fixed dental prosthesis (FDP) typically requires splinting of additional abutments to overcome the loss of bone support of the abutment. Ante's law is used as a guide to plan an FDP with an optimal number of abutments (I). In the glossary of prosthodontics, the Ante's law for FDPs states: "in fixed dental prosthodontics, the combined pericemental area of all abutment teeth supporting a fixed dental prosthesis should be equal to or greater than that of the tooth or teeth to be replaced" (2). Based on this, more abutments may be needed as the edentulous span of an FDP increases (I).

In current practice, most people prefer dental implants as replacement for missing teeth (3). The high survival and success rate of dental implants is widely acknowledged (4-9). Application of biomechanical principles is therefore key to sustain these rates $(5,6,8-12)$.

Well-known theoretical approaches are often not supported by clinical observations (such as the number of dental implants to be used for FDPs) (I3). One common approach is to place a dental implant for each missing tooth (14). From another theoretical perspective, the solution is to place dental implants to support FDP with a central pontic (I5). However, there is a lack of consensus on the optimal number of dental implants required to provide adequate support (5, 7, 16). 
Direct clinical evaluation is the most accurate method to analyze the biomechanical effects of dental implant treatment. However, the complexity of the structures involved makes direct clinical evaluation of the biomechanical behavior of intraosseous structures nearly impossible. The potential difficulties for this type of study include possible ethical issues, applicability of methodology, and time required for the procedure. To overcome these limitations, several studies have employed computational, analytical, and experimental models to evaluate the biomechanics of dental implants. These include finite element analysis (FEA), photoelasticity, and the use of strain gauges $(11,17,18)$.

FEA involves the use of virtual models to simulate and test the progressive resistance and stress distribution of complex structures. This method enables for the investigation of mechanical problems by dividing the problem into many smaller and simpler elements. This approach creates a mesh of elements and solves the problem using mathematical functions. This allows for the simulation and evaluation of the biomechanical behavior of bone, dental implants, and prosthetic component interfaces, which otherwise would be impossible to analyze in vitro or in vivo (II, 17).

The purpose of this study was to evaluate the differences in the distribution and magnitude of stress values in dental implants and tooth abutments of an FDP in identical situations. Furthermore, we sought to investigate the optimal number of dental implants in comparison with the identically placed tooth abutments according to Ante's law. The first null hypothesis stated, "the type of abutment (dental implant and tooth abutments) does not affect the magnitude of stress." The second null hypothesis stated, "the type of abutment does not affect the stress distribution in cortical bone in identical situations." Moreover, the third null hypothesis stated, an "an increase in the number of missing teeth does not affect the distribution and magnitude of stress in tooth abutment models." Finally, the fourth null hypothesis stated, "an increase in the number of missing abutments does not affect the distribution and magnitude of stress in dental implant models."

\section{MATERIAL and METHODS}

We created, homogenized, meshed, and analyzed three-dimensional (3D) models using a computer (Intel Xeon ${ }^{\circledR} \mathrm{R} C P U$ 3,30 GHz processor, $500 \mathrm{~GB}$ hard disk, 14 GB RAM, Windows 7 UItimate Version Service Pack I) with Activity 880 (Smart Optics Sensortechnik GmbH, Sinterstrasse 8, D-44795 Bochum, Germany), computed tomography (CT; ILUMA, Orthocad, CBCT, 3M

\section{Main Points:}

- The increase in the missing tooth resulted with higher stress values in peripheral bone.

- Dental implants and natural tooth abutments had similar stress distribution behaviour.

- The different stress trends were observed in identical treatment plans with dental implants/natural tooth abutments.

- Ante's law may not be suitable for planing dental implants because of the difference in stress trends. Further studies are recommended.
Imtec, OK, USA), Rhinoceros 4.0 (Seattle, WA, USA), 3D-Doctor (Able Software Corp., MA, USA), VRMesh (VirtualGrid, Bellevve, WA, USA), and Algor Fempro (ALGOR, Inc. Pittsburgh, PA, USA).

We constructed a CT data of mandible from a human cadaver using a 3D model of the edentulous mandible with 2-mm cortical bone layer. Then, we transferred the data into the 3D-Doctor and Rhinoceros software in order to generate a 3D finite element model with a l-mm thick slice.

We scanned dental implants, abutments, and dental gypsum models using Activity 880 in order to construct 3D models of teeth and the dental implant-abutment complex. We used a bone-level dental implant (diameter: $4 \mathrm{~mm}$; length: $10 \mathrm{~mm}$ ) for all dental implant-supported models (ISMs). Moreover, we scanned the teeth models for reconstruction according to Wheeler standards using the Rhinoceros software. Then, we reformed the abutment teeth in accordance with tooth preparation principles. We designed superstructure models of FDPs with modified ridge-lap pontics using Wheeler standards as a guide. We also designed frameworks of the restorations with a thickness of $0.3 \mathrm{~mm}$ and a $3-\mathrm{mm}$ connector width of FDPs. Finally, we used cobalt-chromium-supported ceramic as the prosthetic material for the process.

\section{Models}

There were two main model types: teeth-supported models (TSMs) and dental ISMs. We created the first group of models without any missing teeth (TSMO) or dental implants (ISMO). The second group of models comprised of a missing mandibular right first molar and FDPs with supports at the mandibular right second molar and second premolar (teeth-supported, TSMI; dental implant-supported, ISMI). We removed the mandibular right first molar and second premolar and abutments (teeth-supported, TSM2; dental implant-supported, ISM2) at the mandibular right second molar. We then supported the fourunit FDP in the third group of models using the first premolar region. We considered the mandibular right first molar, second premolar, and first premolar missing and the supports of the restoration were at the mandibular right second molar and canine (teeth-supported, TSM3; dental implant-supported, ISM3). In total, we created eight models.

\section{Mesh Creation}

We transferred models created in the Rhinoceros software to Fempro while preserving the 3D coordinates. The models were rigidly solid meshed using bricks and tetrahedral elements. In the bricks and tetrahedral solid modeling system, Fempro uses as many as eight nodes in the model. We used seven-, six-, or fivenode elements when eight-node elements could not achieve the required detail. All the models were linear, homogeneous, and isotropic. Table I displays the properties of the materials used in the study.

\section{Boundary Conditions}

We fixed the models at the base and the mesial and distal edges of the mandible with zero degree of freedom.

\section{Loading Conditions}

Overall, we applied a force of $700 \mathrm{~N}$ to the model by means of 

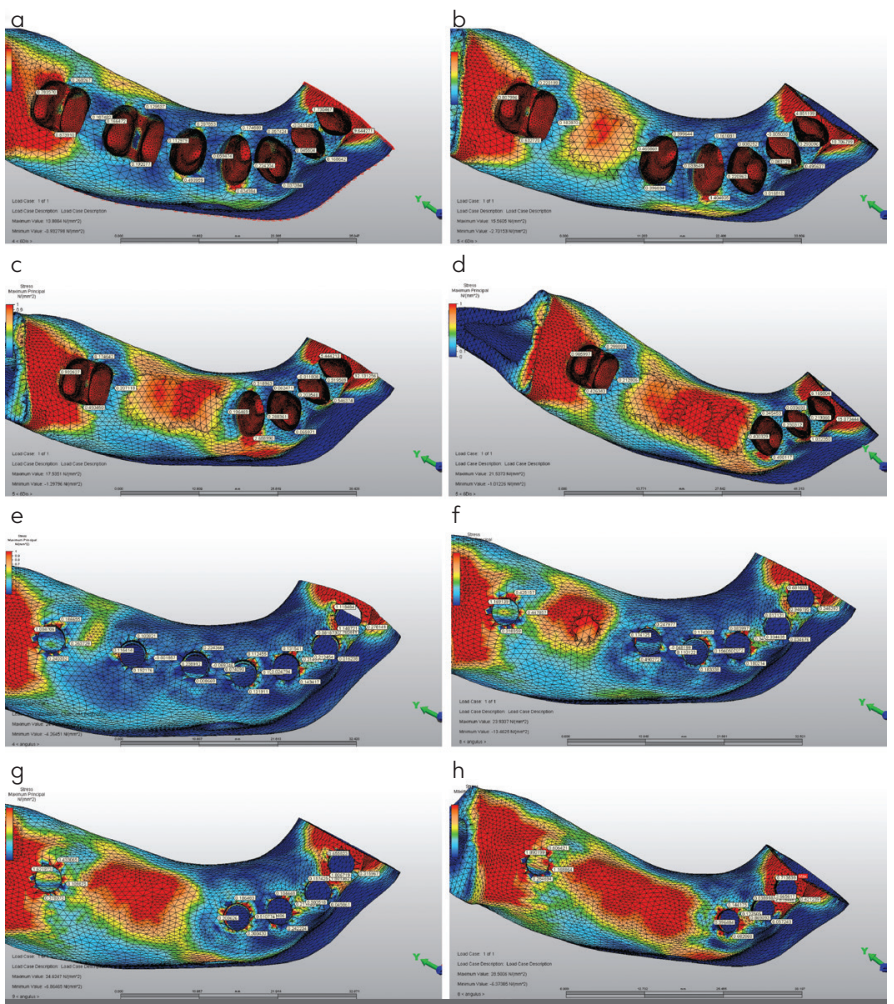

FIGURE I. a-h. Maximum principal stress analysis in the cortical bone of TSM0 (a), TSMI (b), TSM2 (c), TSM3 (d), ISM0 (e), ISMI (f), ISM2 (g), and ISM3 (h). The red color indicates the highest stress distribution area whereas the blue color indicates the lowest stress distribution area. The highest stress values which were used to compare the models are displayed at the left corner of each model

\section{TABLE I. Material properties}

\begin{tabular}{lcc|} 
Materials & Young modulus (MPa) & Poisson ratio \\
\hline Feldspathic porcelain & 82.800 & 0.35 \\
Cobalt-Chromium alloy & 218.000 & 0.33 \\
Enamel & 82.500 & 0.33 \\
Dentin & 18.600 & 0.31 \\
Periodontal ligament & 170 & 0.45 \\
Ti-6Al-4V & 110.000 & 0.35 \\
Cortical bone & 13.700 & 0.3 \\
Trabecular bone & 1.370 & 0.3 \\
\hline
\end{tabular}

seven foodstuffs. Each foodstuff applied $100 \mathrm{~N}$ of vertical force to each tooth's occlusal surface. We used TSMO and ISMO as control groups. We used the highest maximum principal stress values (omax) and minimum principal stress values (omin) in the cortical bone for comparison. We also used these values to calculate the ratio between identical situations or similar supporting-abutment types. We considered a more than 10\% difference in the values as clinically important. There was no need for any statistical method due to the nature of our study.

\section{RESULTS}

Figure I-3 present the results of FEA. Figure I-2 demonstrate the maximum and minimum principal stress analysis, respectively. Figure 3 shows a graphical representation of the highest stress values. The positive values in the graphical illustrations reflect
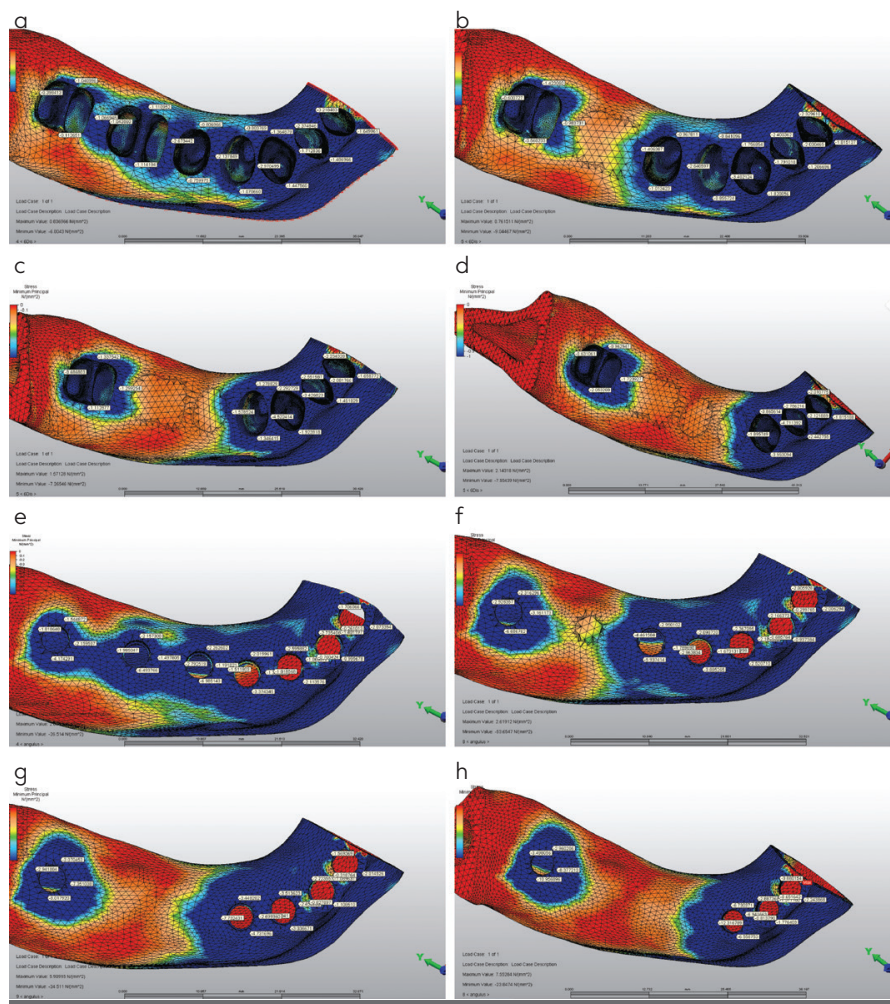

FIGURE 2. a-h. Minimum principal stress analysis in the cortical bone of TSM0 (a), TSMI (b), TSM2 (c), and TSM3 (d), ISM0 (e), ISMI (f),

ISM2 (g), and ISM3 (h). The blue color indicates the highest stress distribution area, whereas the red color indicates the lowest stress distribution area. The highest stress values which were used to compare the models are displayed at the left corner of each model

the omax values (Figure 3a), whereas the negative values reflect the omin values (Figure $3 \mathrm{~b}$ ). The red color in Figure I and the blue color in Figure 2 indicate the highest stress distribution area. The left lower corner of each figure displayed the highest stress value of the models. We then used these values to compare the models.

In general, ISMs resulted in higher stress values than TSMs. ISM models had $32 \%-80 \%$ higher omax stress values and $181 \%-342 \%$ higher omin values than their corresponding TSMs. The distribution of stress in the cortical bone was similar in identical models regardless of the abutment type. In TSMO and ISMO, stress was well-distributed. However, the loss of tooth/teeth resulted in more stress concentration at the edentate (pontic) area.

The omax and omin values in the cortical bone increased with increase in the number of missing teeth. TSMI, TSM2, and TSM3 showed higher omax stress values (12\%, 26\%, and 55\%, respectively) (Figure lb-d) and higher omin stress values (51\%, $21 \%$, and $26 \%$, respectively) (Figure $2 \mathrm{~b}-\mathrm{d}$ ) than TSM0 (Figure la, 2a). We observed similar omax and omin stress values in ISMO (Figure IE and 2E), ISMI (Figure If, 2f), and ISM2 (Figure Ig, 2g). Meanwhile, ISM3 showed $14 \%$ higher omax stress value (Figure $\mathrm{lh}$ ) and $10 \%$ lower omin stress value (Figure $2 \mathrm{~h}$ ) than the others (Figure $2 \mathrm{e}-\mathrm{g}$ ).

\section{DISCUSSION}

We rejected the first null hypothesis based on the differences in stress values between the numbers of tooth abutments and dental implants in the different combinations of the posterior 
A

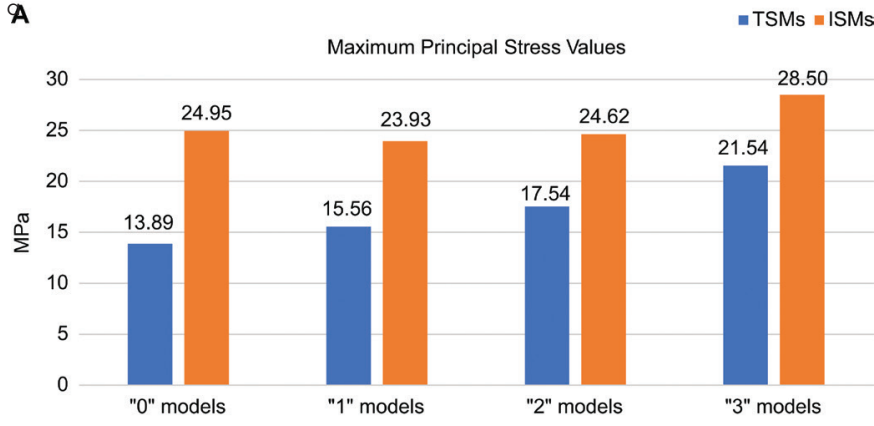

B

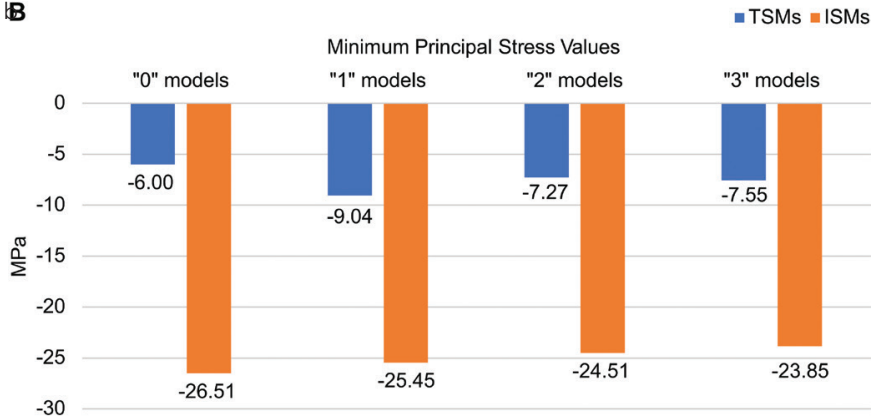

FIGURE 3. a, b. Graphical illustration of the maximum (a) and minimum (b) principal stress values in the cortical bone of TSMs and ISMs. The positive values reflect the omax values, whereas the negative values reflect the omin values

edentulism. We accepted the second null hypothesis was accepted based on the similar stress distribution in the identical models. We rejected the third null hypothesis based on the differences between TSMs with respect to stress distribution and levels of omax and omin. We partially accepted the fourth null hypothesis based on the similarity of the stress distribution and magnitude of omax and omin among ISMs, with the exception of the stress values of ISM3.

According to the results, the magnitude of stress depends not only on the number but also on the type of abutment (tooth or dental implant). Dental implants increase the stress values in the cortical bone and were compared with tooth abutments. An increase in the number of missing teeth in an FDP may also lead to higher stress values in the cortical bone regardless of the abutment type (dental implant or tooth). Reduced number of dental implants can be compensated by increasing the number of dental implants. (19). The diameter and length of dental implants were constant in the present study for the standardization of ISMs.

In vivo and in vitro methods for stress analyses of living tissues, such as bones, teeth, and periodontium, are typically challenging (and sometimes even impossible) (II, I7, 18). Each method has advantages and limitations (17). FEA is a suitable method for stress analysis of structures with complex geometry (II, 17). We evaluated the stress in all models with FEA in the present study as stipulated by literature.

Ismail et al. (20) compared two-dimensional (2D) and 3D FEA and reported that 2D analysis was sufficient to assess principal stress distribution. However, the 2D method did not reflect the normal stress distribution in detail. In another study, minor differ- ences were observed between 2D and 3D analyses. The authors opined that the 3D model may provide a better geometrical representation (2I). Meijer et al. (22) reported that a 3D model of the region to be examined is sufficient and less time-consuming compared with creating a model of the entire mandible. In this study, we preferred the 3D FEA and we used a 3D image of the working region rather than modeling the entire mandible to obtain realistic models and results.

The maximum occlusal force during mastication varies with natural dentition and dental implants due to muscle size, shape of bones/temporomandibular joint tissues, and the extent of jaw separation $(23,24)$. Furthermore, factors such as the bite direction and sex of the patient are known to affect the maximum bite force $(24,25)$. Paphangkorakit and Osborn (24) reported that the maximum occlusal force in the anterior region is in the range of 90-307 N, whereas Waltimo and Könönen (25) reported that the maximum physiologic occlusal force in the mandibular right molar region is approximately $300 \mathrm{~N}$. In a study by Haraldson et al. (26), the median value of the maximal force in dental implants was $143.5 \mathrm{~N}$. In the present study, we applied a static $100 \mathrm{~N}$ load for each tooth with a food-shaped model, which is within the reported physiological limits. This kind of loading may present a more realistic load distribution, thanks to the replication of food-shaped model.

We used principal stress (compressive and tensile stress) values to evaluate brittle materials such as bones. Failure occurs if the compression stress in bone is equal to or exceeds the highest compression stress. Thus, principal stress allows evaluation by determining the difference between the tensile and compressive stress (27). Hence, we used principal stress in this study for the analysis of stress values in bone.

A comparison of the natural tooth with the dental implant revealed that an intrusion in natural dentition occurs during mastication, and stress accumulates around the dental implant. Overload may impose high stress on the supporting bone, which leads to bone resorption. The direct opposite of this phenomenon may result in disuse atrophy of bone (28). Most FEA studies evaluate stress levels, implying that lower stress represents a more favorable result. However, in the present study, we evaluated the magnitude and distribution of stress only.

The highest tensile strength of the cortical bone as I2I MPa and the maximum compression strength was reported as $167 \mathrm{MPa}$ (29). In this study, the highest tensile stress value was $29 \mathrm{MPa}$ in the cortical bone of ISM3. This value is lower than the highest tensile strength of bone. The highest compression stress value was $27 \mathrm{MPa}$ in the cortical bone of ISM0. This value is lower than the maximum compression strength of bone.

In a systematic review, Heydecke et al. (7) evaluated the survival rates with regard to the required number of dental implants. They reported that a dental implant-to-replaced-units-ratio of 2/3 can be considered adequate. However, for full-arch dental implant-supported FDPs, the corresponding ratio is between $\mathrm{I} / 3$ and $\mathrm{I} / 2$, which is lower than the first calculated ratio. In present study, we compared the ISMs with identical tooth-supported planning by means of stress transmission to the peripheral bone, and dental implants transmitted higher 
stress values to bone. The maximum-stress-ratio (ISM/identical TSM) was between 1.32 and 1.80 for maximum principal stress value and between 2.81 and 4.42 for minimum principal stress values in the cortical bone. This ratio is not a dental implant-to-replaced-units ratio; however, it seems plausible that dental implants present higher stress on the bone than tooth abutments, and the importance of avoiding overload is a key consideration during planning of dental implant treatment. The trends in the change of stress values differed according to types of abutments in identical situations. These findings reveal that the behavior of dental implants is different from that of tooth abutments in supporting an FDP. Furthermore, these findings also revealed that it is not suitable to use Ante's law in the planning of dental implants. We therefore recommend further studies to determine the biomechanical conditions of dental implant treatment.

To the authors' knowledge, this was the first study comparing the stress distribution in natural teeth and dental implants in identical conditions by using Ante's law. Undoubtedly, the current study has some limitations. In dentistry, 3D FEA models have been widely used to study the biomechanics of loaded stress. However, in the current study, use of the FEA program was limited by unrealistic assumptions such as homogeneous, linear elastic, and isotropic conditions for the bone, tooth, and periodontal ligament. The design and material of the dental implant and prosthesis were constant to simplify the models. It should be kept in mind that the variations in type of material, diameter, length, angulation, and surface treatment of dental implants and the design and material of the prosthesis can affect the stress in peripheral bone $(9,23,30)$. Furthermore, we assumed that the bonding of the bone and dental implant was perfect and that the masticatory forces were static and loaded axially using a suitable model (fitting for the foodstuff in the occlusal surface of the crowns) as compared to the dynamic masticatory forces, (oblique to the occlusal surface). Consequently, the reconstruction did not replicate all the natural details. Mathematical models can be used only to explain experimental results, and in science, their predictive power is used for comparisons (18). In the present study, we evaluated the distribution and magnitude of stress in identical edentulous treatment plans with different types of abutments. However, the numbers and distribution of abutments were not changed. Although we could not replicate the natural stress values because of these limitations, we observed differences with respect to the magnitude and distribution of stress between differently planned edentulism models. We recommend further studies to understand more about the biomechanical needs for bone stimulation and the effects of other variables on stress distribution of tooth and dental implant supports.

Within the limitations of this finite element study, dental ISMs showed higher stress values than TSMs. The increase in the number of missing tooth enhanced the stress in peripheral bone for both abutment types (tooth or dental implant). We observed a similar behavior with respect to the distribution of stress with dental implant and natural tooth abutments supporting partial FDP. However, we recorded different trends with respect to the change in the magnitude of stress with increase in the number of missing teeth in identical treatment plans. We considered the importance of avoiding overload in dental implant planning because of the higher stress values occurring in the peripheral bone of ISMs. Our results reveal that it may not be suitable to use Ante's law to plan dental implants because of different trends of change in the magnitude of stress in identical treatment plans, even if the distribution of stress was similar. We recommend further studies to understand more about the biomechanical effects of the number and distribution of dental implants and to determine the biomechanical differences between dental implants and tooth abutments.

\section{Ethics Committee Approval: N/A}

\section{Informed Consent: N/A}

Peer-review: Externally peer-reviewed.

Author contributions: Concept - M.M.U., H.A., A.N.O.; Design - H.A.; Supervision - M.M.U., A.N.O.; Resource - M.M.U, H.A., A.N.O.; Materials M.M.U., H.A.; Data Collection and/or Processing - H.A.; Analysis and/ or Interpretation - M.M.U., H.A., A.N.O.; Literature Search - H.A.; Writing - H.A.; Critical Reviews - M.M.U., A.N.O.

Acknowledgements: The authors would like to thank Ayberk Yağız for Finite Element Anaylsis.

Conflict of Interest: Authors have no conflicts of interest to declare.

Financial Disclosure: The study was supported by a grant from the Near East University Centre of Excellence (grant numbers 2016-0400I, CE7052016).

\section{REFERENCES}

I. Chitumalla R, Munaga S, Khare A, Agarwal S, Bhoyar A, Parlani S. Stress distribution among periodontally compromised abutments: A comparative study using three-dimensional finite element analysis. Contemp Clin Dent 2012; 3(4): 452-8. [Crossref]

2. Driscoll CF, Freilich MA, Guckes AD, Knoernschild KL, Mcgarry TJ. The Glossary of Prosthodontic Terms: Ninth Edition. J Prosthet Dent 2017; II7(5S): el-105. [Crossref]

3. Leung KCM, McGrath CPJ. Willingness to pay for implant therapy: a study of patient preference. Clin Oral Implants Res 20I0; 21(8): 78993. [Crossref]

4. De Kok P, Kleverlaan CJ, De Jager N, Kuijs R, Feilzer AJ. Mechanical performance of implant-supported posterior crowns. J Prosthet Dent 2015; ||4(I): 59-66. [Crossref]

5. Thalji G, Bryington M, De Kok IJ, Cooper LF. Prosthodontic Management of Implant Therapy. Dent Clin North Am 2014; 58(I): 207-25. [Crossref]

6. Sun HL, Huang C, Wu YR, Shi B. Failure rates of short $(\leq 10 \mathrm{~mm})$ dental implants and factors influencing their failure: a systematic review. Int J Oral Maxillofac Implants 20II; 26(4): 816-25.

7. Heydecke G, Zwahlen M, Nicol A, Nisand D, Payer M, Renouard F et al. What is the optimal number of implants for fixed reconstructions: a systematic review. Clin Oral Implants Res 2012; 23(Suppl 6): 217-28. [Crossref]

8. Papaspyridakos P, Chen C-J, Chuang S-K, Weber H-P. Implant loading protocols for edentulous patients with fixed prostheses: a systematic review and meta-analysis. Int J Oral Maxillofac Implants 2014; 29(Suppl): 256-70. [Crossref]

9. Ogawa T, Dhaliwal S, Naert I, Mine A, Kronstrom M, Sasaki K et al. Impact of implant number, distribution and prosthesis material on loading on implants supporting fixed prostheses. J Oral Rehabil 20I0; 37(7): 525-31. [Crossref]

10. Sahin S, Cehreli MC, Yalçin E. The influence of functional forces on the biomechanics of implant-supported prostheses--a review. J Dent 2002; 30 (7-8): 27I-82. [Crossref] 
II. Geng JP, Tan KBC, Liu GR. Application of finite element analysis in implant dentistry: a review of the literature. J Prosthet Dent 200I; 85(6): 585-98. [Crossref]

12. Stoumpis C, Kohal R-J. To splint or not to splint oral implants in the implant-supported overdenture therapy? A systematic literature review. J Oral Rehabil 20II; 38(II): 857-69. [Crossref]

13. Taylor TD, Agar JR, Vogiatzi T. Implant prosthodontics: current perspective and future directions. Int J Oral Maxillofac Implants 2000; I5(I): 66-75.

14. Rangert B, Jemt T, Jörneus L. Forces and moments on Branemark implants. Int J Oral Maxillofac Implants 1989; 4(3): 24I-7.

15. Buser D, Belser UC, Lang NP. The original one-stage dental implant system and its clinical application. Periodontol 2000 1998; 17: 106-18. [Crossref]

16. Brunski JB. Biomechanical aspects of the optimal number of implants to carry a cross-arch full restoration. Eur J Oral Implantol 2014; 7(Suppl 2): SIII-3I.

17. Pesqueira A, Goiato M, Gennari-Filho H, Monteiro D, Dos Santos D, Haddad M, et al. The use of stress analysis methods to evaluate the biomechanics of oral rehabilitation with implants. J Oral Implantol 2012; 40(2): 217-28. [Crossref]

18. Guven S, Beydemir K, Dundar S, Eratilla V. Evaluation of stress distributions in peri-implant and periodontal bone tissues in 3- and 5-unit tooth and implant-supported fixed zirconia restorations by finite elements analysis. Eur J Dent 2015; 9(3): 329-39. [Crossref]

19. Anitua E, Tapia R, Luzuriaga F, Orive G. Influence of implant length, diameter, and geometry on stress distribution: a finite element analysis. Int J Periodontics Restorative Dent 20I0; 30(I): 89-95.

20. Ismail YH, Pahountis LN, Fleming JF. Comparison of two-dimensional and three-dimensional finite element analysis of a blade implant. Int J Oral Implantol 1987; 4(2): 25-31.
21. Romeed SA, Fok SL, Wilson NHF. A comparison of 2D and 3D finite element analysis of a restored tooth. J Oral Rehabil 2006; 33(3): 209-15. [Crossref]

22. Meijer HJ, Starmans FJ, Bosman F, Steen WH. A comparison of three finite element models of an edentulous mandible provided with implants. J Oral Rehabil 1993; 20(2): 147-57. [Crossref]

23. Lee J-I, Lee Y, Kim Y-L, Cho H-W. Effect of implant number and distribution on load transfer in implant-supported partial fixed dental prostheses for the anterior maxilla: A photoelastic stress analysis study. J Prosthet Dent 2016; II5(2): 161-9. [Crossref]

24. Paphangkorakit J, Osborn JW. The effect of pressure on a maximum incisal bite force in man. Arch Oral Biol 1997; 42(I): II-7. [Crossref]

25. Waltimo A, Könönen M. A novel bite force recorder and maximal isometric bite force values for healthy young adults. Scand J Dent Res 1993; I0I(3): 17I-5. [Crossref]

26. Haraldson T, Carlsson GE, Ingervall B. Functional state, bite force and postural muscle activity in patients with osseointegrated oral implant bridges. Acta Odontol Scand 1979; 37(4): 195-206. [Crossref]

27. Akça K, Iplikçioğlu H. Finite element stress analysis of the influence of staggered versus straight placement of dental implants. Int $\mathrm{J}$ Oral Maxillofac Implants 200I; 16(5): 722-30.

28. Alper B, Gultekin P, Yalci S. Application of Finite Element Analysis in Implant Dentistry. In: Ebrahimi F, editor. Finite Element Analysis New Trends and Developments. InTech 2012. [Crossref]

29. Brunski JB, Puleo D a, Nanci A. Biomaterials and biomechanics of oral and maxillofacial implants: current status and future developments. Int J Oral Maxillofac Implants 2000; I5(I): 15-46.

30. Iplikçioğlu H, Akça K. Comparative evaluation of the effect of diameter, length and number of implants supporting three-unit fixed partial prostheses on stress distribution in the bone. J Dent 2002; 30(I): 4l-6. [Crossref] 